\title{
Sharing experiences with comprehensive responses to adolescent reproductive health needs in Africa
}

\author{
Nafissatou J. Diop \\ Population Council \\ Anta Fall Diagne \\ Population Council
}

Follow this and additional works at: https://knowledgecommons.popcouncil.org/departments_sbsr-rh

Part of the Demography, Population, and Ecology Commons, Health Services Research Commons, and the International Public Health Commons

How does access to this work benefit you? Let us know!

\section{Recommended Citation}

Diop, Nafissatou J. and Anta Fall Diagne. 2008. "Sharing experiences with comprehensive responses to adolescent reproductive health needs in Africa," FRONTIERS Final Report. Washington, DC: Population Council. 


\title{
Sharing Experiences with Comprehensive Responses to Adolescent Reproductive Health Needs in Africa
}

\author{
Nafissatou J. Diop \\ Anta Fall Diagne
}

March 2008

This study was made possible through the generous support of the people of the United States through the United States Agency for International Development (USAID), under the terms of Cooperative Agreement No. HRNA-00-98-00012-00 and In-house project 5800 53126. The contents of this report are the responsibility of the FRONTIERS Program and do not necessarily reflect the points of view of USAID or the United States Government. 


\section{TABLE OF CONTENTS / TABLE DES MATIERES}

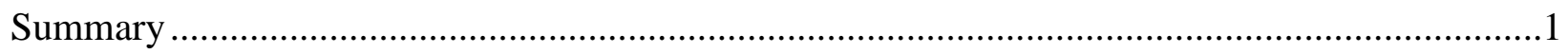

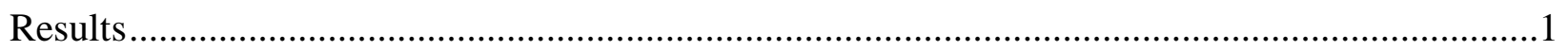

Conclusion

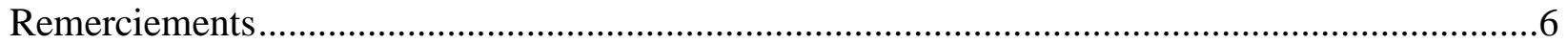

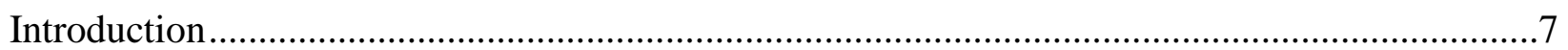

Leçons apprises de l'étude de Frontières sur l'Amélioration de la SRA au Sénégal ....................7

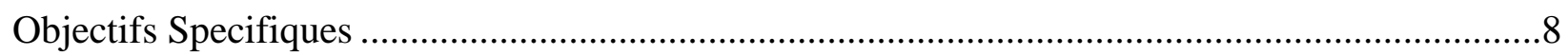

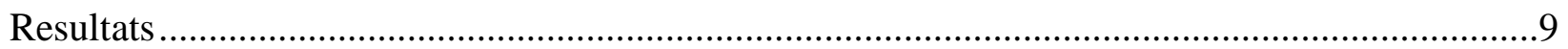

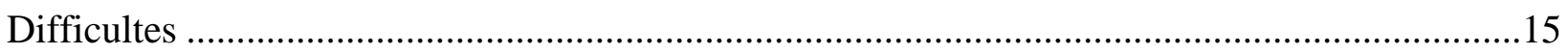

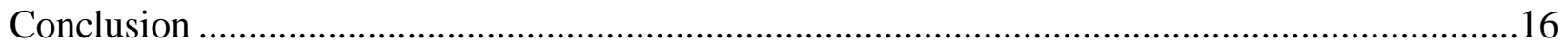

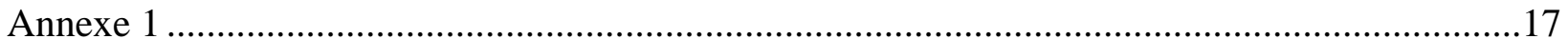




\begin{tabular}{ll} 
Abbreviations and Acronyms / abréviations et acronymes \\
AEMO & Action Educative en Milieu Ouvert \\
ASBEF & Association Sénégalaise pour le Bien-Être Familial \\
APADOC & Alliance of Parents, Adolescents and the Community \\
AYA & Alliance Africaine des Jeunes \\
BAD & Banque Africaine de Développement \\
CCC & Communication pour un Changement de comportement \\
CEFOREP & Centre de Formation et de Recherche en Santé de la Reproduction \\
CNLS & Conseil National de Lutte contre le SIDA \\
DLSI & Division de Lutte contre le SIDA \\
DSR & Division de la Santé de la Reproduction \\
GEEP & Groupe pour l'Etude et l'Enseignement de la Population \\
IPPF & International Planned Parenthood Federation \\
OCB & Organisation Communautaire de Base \\
OMS & Organisation Mondiale de la Santé \\
ONG & Organisation Non Gouvernementale \\
RO & Recherche Opérationnelle \\
SCS & Save The Children Suède \\
SNIS & Système National d'Information Sanitaire \\
SNSA & Stratégie Nationale en Santé des Adolescents \\
SRA & Santé de la Reproduction des Adolescents \\
SSCA & Services de Santé Conviviaux pour les Adolescents \\
UNFPA & Fonds des Nations Unis pour la Population \\
UNICEF & Fonds des Nations Unis pour l'Enfance \\
USAID & United States Agency for International Development \\
YMCA & Young Mens Christian Association \\
\hline
\end{tabular}




\section{Summary}

In 1999, the Population Council's USAID-funded Frontiers in Reproductive Health (FRONTIERS) project, began a three-year collaboration in Senegal with the Ministries of Health $(\mathrm{MOH})$, Education (MOE), and Youth (MOY), the Centre de Formation et de Recherche en Santé de la Reproduction (CEFOREP), the Groupe pour l'Etude et l'Enseignement de la Population (GEEP) and the World Health Organization (WHO), to develop and test the feasibility, effectiveness and cost of a multisectoral model comprising school-, clinic- and community-based interventions to improve the reproductive health of girls and boys aged 10-19. This adolescent reproductive health (ARH) pilot project showed that a multisectoral, multidisciplinary approach can increase knowledge, enhance attitudes, and foster positive behaviors for reproductive health among young people, the community, schools and health care providers. These findings prompted a series of scaling-up activities, launched in 2004, to create a favorable policy and funding environment for expanding these ARH interventions and institutionalizing ARH programs in several districts. Following requests from several neighboring countries, and given the long collaboration with WHO, FRONTIERS initiated efforts in 2007 to enable utilization of these findings in other African countries.

The purpose is to promote the utilization of multisectoral approaches for improving adolescent reproductive health programming by governments, donors, national and international agencies in francophone West Africa. Specifically, the project will:

- Communicate lessons learned about multisectoral approaches and tools for youth programming in sub-Saharan Africa at a regional workshop

- Develop a consensus on successful strategic directions for youth programming in West Africa

- Build the capacity of service delivery institutions in implementing multisectoral interventions to improve adolescent reproductive health in francophone West Africa

This project addressed USAID's strategic objectives on investing in people, and FRONTIERS intermediate result on demonstrating global leadership in policy, advocacy and services and support to the field to implement effective and sustainable FP/RH programs. It also addressed WHO's objective of sharing evidence-based practices.

\section{Results}

\section{Developing a partnership for a Regional Youth Consultative Workshop on community interventions and influencing policies and programs}

In partnership with WHO AFRO, a three-day regional workshop on community-based ARH interventions for adolescent and youth programs was held in Accra, Ghana in February 2008. Participants included representatives from the ministries of health, education, and youth, NGOs and Youth Associations in Burkina Faso, Ghana, Kenya, Mauritania, Mozambique, Namibia, Senegal, Tanzania, and Zimbabwe, and 31 program managers from WHO/Geneva, WHO/AFRO, UNICEF, UNFPA, Save the Children, and the London School of Hygiene and Tropical Medicine. FRONTIERS supported the participation of representatives from Senegal, Kenya, Burkina Faso and Mauritania. 
FRONTIERS shared lessons learned from the experiences in Senegal and Kenya to introduce and scale-up adolescent services within government programs during the pilot phase and the subsequent scale-up process. Experiences from the UNFPA-supported African Youth Alliance (AYA) project and the WHO-supported Alliance of Parents, Adolescents and the Community (APADOC) program contributed to the presentation of a broad range of multisectoral approaches for addressing adolescent reproductive health. All three programs have in common a holistic and strategic approach, building on existing institutions and organizations. All experiences highlighted some common challenges:

- Education about condoms and contraceptives remains very sensitive, even in countries with a high prevalence of HIV/AIDS.

- Quality assurance of the interventions during scaling up was problematic.

- Maintaining the focus of the interventions on increased use of health services by adolescents was challenging.

- Lack of indicators for demonstrating increased community support and increased demand for services by adolescents.

A conceptual framework was drafted in order to clarify the important links that need to be established between community interventions and the use of services by adolescents.

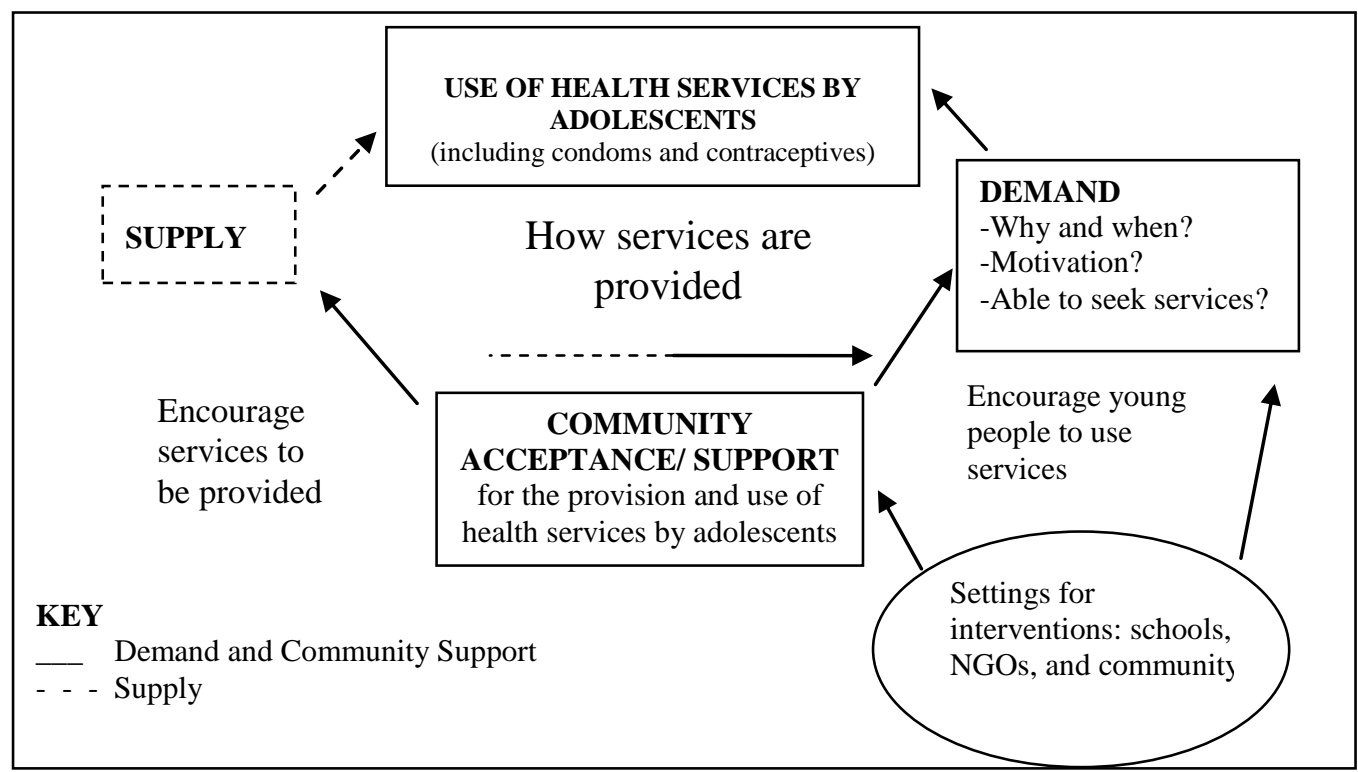

Some indictors that could be used were proposed:

\section{Demand for health services by adolescents}

- Adolescents know when and why health services should be used (knowledge)

- Adolescents know where health services can be obtained; what the conditions are for their use (knowledge)

- Adolescents state intentions to use services, if needed (attitude)

- Adolescents use or report use of services (behavior) 
Community acceptance and support of service provision and use

- Awareness of adolescents' need for health services (knowledge)

- Approval of service provision and use (attitude)

- Actions to improve service provision to adolescents (e.g., advocacy in the community) (behavior)

- Actions to improve service use by adolescents (e.g., accompanying adolescents to services; providing funds for fees and transport to services) (behavior).

\section{Developing an orientation guide for adolescent reproductive health programming}

Based on the lessons learned and tools developed and validated within the three programs presented, the outline and format for a generic strategic orientation guide for implementing multisectoral approaches to address adolescent reproductive health was developed during the workshop. The outline developed during the working groups is being summarized by a small technical team composed of staff from WHO, UNFPA and FRONTIERS with the support from a WHO consultant. The objective of this guide is to highlight key elements of multisectoral adolescent health programming that can be used by ARH managers in various sectors working together at the country level. Facilitating and inhibiting factors have been identified and synthesized for institutional, programmatic, and policy efforts at the national and district level. The guide is being disseminated to program managers and development partners throughout the region.

\section{Building the capacity of service delivery institutions in Francophone countries}

In addition to this regional programming effort, FRONTIERS supported capacity building activities in two francophone countries that had demonstrated interest in prioritizing ARH issues and that had previously received technical assistance from FRONTIERS to adapt and introduce the Senegal model.

\section{Mauritania}

The Mauritanian Ministry of Education requested permission from FRONTIERS to adapt and translate the Grandir en Harmonie curriculum into Arabic, with financial support from UNICEF and UNFPA. FRONTIERS provided technical assistance for a validation workshop and a Training of Trainers session for youth educators; UNICEF and UNFPA supported the workshop and identified the Mauritanian trainees with the Ministry. FRONTIERS supported the trainers' technical assistance during two weeks. A total of 44 trainees and more than 200 peer educators are currently working in Mauritania following this training.

\section{Burkina Faso}

The MOH in Burkina Faso has already taken leadership in responding to adolescent needs. They expressed a strong interest in the Senegal experience and tools, following a presentation at a regional workshop in June 2005. Additionally, it was agreed that the Senegalese National Strategy on Adolescent Health policy document could provide a useful model for Burkina Faso and $\mathrm{WHO}$ has also developed standards for youth-friendly services in Burkina Faso.

Unfortunately to date, only the tools have been shared with $\mathrm{MOH}$ program managers. 


\section{Providing technical assistance for training and facilitating the exchange of trainers}

Senegal has already benefited from holding several 'training of trainers' exercises, with both the curriculum entitled Grandir en Harmonie and the Orientation of Health Providers curriculum. This has created a core group of more than 200 'master trainers' that is now available and could be used in other francophone countries. Master trainers are staff responsible at the national and local levels for the training of those staff that plan to use either of the two curricula. They include training staff from the MOY, MOH, Ministry of Social Development and Family, youth center trainers, representatives of important youth associations, and public health school trainers.

FRONTIERS facilitated contact with the Mauritanian group and supported the negotiation between the Senegal MOY and the Mauritania MOE for the provision of technical assistance and travel of three trained staff from Senegal.

A similar experience was developed with the YMCA which requested capacity building assistance from FRONTIERS. The YMCA used funding provided by the World Bank through the National AIDS Council to conduct ARH activities among 200 youth CBOs throughout Senegal, for which FRONTIERS facilitated the provision of trainers. To date, the YMCA has reached 8,000 youth (5,693 girls and 2,626 boys) with the Grandir en Harmonie curriculum.

Save the Children/Sweden (SCS) is using the youth curriculum in five West African countries for its AIDS prevention program. Given the impact of HIV/AIDS on children's lives and the infection rate among youth, SCS decided to integrate HIV/AIDS issues into its regional program in 2004. Within its framework, SCS identified NGOs in Togo, Burkina Faso, Senegal, Guinea and Côte d'Ivoire that work with adolescents aged between 10 and 18 years, schooled or unschooled, from both rural and urban areas.

\section{Sharing of resource packages of key materials}

FRONTIERS has compiled substantial experience and developed materials for francophone audiences. After extensive review, editing and printing, an attractive package has been distributed to partners at the national and district levels throughout the region comprising: 1,000 copies of the leaflet "Savoir pour Agir"; 1,000 copies of the notebook "carnet pour une bonne conduite"; 300 CD ROMs of the curriculum Orientation for Health Providers in Adolescent Health; 100 folders on Adolescent Health with summaries on evidence-based youth programs, scaling up interventions, documentation of process, policy document, and achievements. A poster in French and in English was also produced for exhibitions.

\section{Increasing attention among political leaders, program managers and the public to adolescent reproductive health issues through the media}

A set of radio programs was organized with the West African Democracy Radio (WADR). WADR was created by the Open Society Initiative for West Africa (OSIWA) that also seeks to offer West Africans a medium of exchanging information among themselves and establishing an interaction with the rest of the world. WADR is a trans-national radio station covering all West African countries, including Chad, Cameroon and Mauritania, with the aim of becoming the hub for a network of public, private and community radios across the subregion. The programs described adolescent reproductive health and development strategies through interviews with staff from the Senegalese ministries, development partners, and community members. 
Adolescents themselves are invited to exchange perspectives on adolescent issues during three weekly radio programs named: Capital Health (on Monday), Fifty-Fifty (on Wednesday) and Youth Connection (on Friday).

\section{Conclusion}

This project was able to effectively reach and influence policies at international institutions such as WHO, UNFPA and Save the Children. Several countries also benefited through a regional partnership. Thorough documentation of lessons learned and the process of dissemination were able to increase program managers' and the public's awareness of the necessity and efficiency of a multisectoral ARH approach.

Although feasible, there are also challenges in replicating any model to other countries because of contextual factors. For example, the social context of Mauritania slowed the ARH program's adoption process of the multisectoral approach. In some countries, such as Burkina Faso, there was a problem of poor leadership and a lack of response by government sectors. Scaling up interventions requires all stakeholders being involved in the planning, implementation and review processes.

The slow pace of implementation of joint activities with WHO, the maintenance of sensitive sessions on sexuality during the Arabic translation of the curriculum, and the lack of sufficient funding at the country level to replicate interventions were the most challenging elements.

The most important recommendations for those seeking to replicate this approach to maximizing utilization of findings from research and scaling up effective service models are the continued involvement of key institutions from the outset, the use of a participatory process to ensure ownership and build capacity, and widespread and repeated dissemination of the results expressed as programmatic recommendations. 


\section{REMERCIEMENTS}

Tous nos remerciements vont à l'encontre de l'USAID qui a bien voulu accepter de soutenir le processus d'utilisation et d'extension du modèle de la recherche opérationnelle et des outils issus de cette recherche.

L'équipe adresse aussi ses vifs remerciements à toutes les personnes qui se sont investies dans la réalisation de ce travail. Leur engagement a contribué à son succès. Il s'agit d'abord du Ministère de la Santé et de la Prévention à travers sa Division de la Santé de la Reproduction, ses équipes cadres de Région et de Districts et aussi le Ministère de la Jeunesse, de la Femme, de la Famille et du Développement Social, et de l'Education.

L'exécution du projet a été facilitée par l'appui et la disponibilité d'institutions et de personnes qui ont composé le Comité restreint chargé du suivi des recommandations des activités de santé des adolescents.

Ce projet a aussi bénéficié de l'appui d'experts dans le domaine de la santé et du développement des adolescent(e)s/Jeunes.

Cette liste n'est certainement pas exhaustive, plusieurs autres personnes ayant apporté une précieuse contribution pour la réalisation de ce travail. Qu'elles trouvent ici l'expression de notre sincère reconnaissance. 


\section{Introduction}

En 1999, FRONTIERS a entamé une collaboration de 3 ans avec l'Organisation Mondiale de la Santé (OMS), les Ministères de la Santé, de l'Education, de la Jeunesse, le Centre de Formation et de Recherche en Population et le Groupe pour l'Etude et l'Enseignement de la Population afin de tester la faisabilité, l'efficacité et les coûts d'interventions au niveau des écoles, des structures de santé et de la communauté pour améliorer la Santé de la Reproduction des Adolescents (SRA) âgés de 10 à 19 ans.

Cette étude sur la SRA a montré qu'une approche multisectorielle et multidisciplinaire permet d'augmenter les connaissances, les attitudes et les comportements responsables pour une santé de la reproduction parmi les jeunes, la communauté, les écoles et le personnel de santé.

Ces résultats positifs ont encouragé les responsables de programmes à maintenir et à étendre les activités envers la santé des adolescents. C'est ainsi qu'en 2004, l'USAID a accepté de financer un projet d'utilisation des résultats de la recherche opérationnelle. FRONTIERS a fourni une assistance technique aux Ministères de la Santé, de l'Education, de la Jeunesse, des Sports et de la Justice afin de renforcer les capacités à élaborer une politique et à mettre en œuvre un programme multisectoriel en SRA.

\section{Leçons apprises de l'étude de Frontières sur l'Amélioration de la SRA au Sénégal}

\section{- Approche Multisectorielle}

L'implication directe des agences gouvernementales et des secteurs communautaires a établi l'importance de la SRA en tant que cadre social et sujet politique. L'engagement fort de l'OMS a donné une plus grande impulsion à cette approche multisectorielle

\section{- Recherche visant les besoins locaux}

Les résultats de la recherche opérationnelle ont été les éléments clés pour maintenir les activités de SRA dans la mesure où elles étaient bâties sur une méthode de recherche scientifique. En outre, le projet est venu répondre aux besoins locaux : Les décideurs politiques, les communautés et les parents ont pris conscience de la nécessité de prendre en charge la question de la SRA, ce qui a facilité un engagement fort.

\section{- Utilisation des institutions existantes}

Une des lignes directrices de l'étude sur la SRA était de bâtir la pérennité à travers les institutions existantes plutôt que d'en créer des nouvelles. Par exemple, le partenariat entre les associations de jeunes et les structures de santé publique a mené à un niveau d'engagement inhabituel et au maintien des pairs éducateurs dans le programme. Les pairs éducateurs qui avaient été formés durant la recherche opérationnelle ont mis en place un système de formation de nouveaux groupes de pairs éducateurs.

\section{- Dissémination continue des résultats}

En plus des disséminations au niveau central, des ateliers de dissémination se sont tenus dans 9 régions au Sénégal. Les résultats de la Recherche Opérationnelle sur l'Amélioration de la Santé de la Reproduction des Adolescents au Sénégal, de même que les documents de politiques en 
SRA ont été largement partagés avec les responsables de programmes de santé, de jeunesse et de l'éducation des régions et des districts.

Des outils de formation et de Communication pour un Changement de Comportement (CCC) ont été validés par les autorités et largement partagés lors de différentes rencontres sur le plan national ou dans la sous région.

\section{- Investissement à long terme}

Prendre en charge la santé de la reproduction des adolescents nécessite que des changements politiques et sociaux importants interviennent sur une période de plusieurs années. Un élément majeur dans ce processus d'utilisation et de pérennisation est le support financier continu de l'USAID, à travers l'assistance technique de FRONTIERS. Cela s'est récemment concrétiser par des financements accordés à la DSR par la Banque Mondiale à travers les fonds de lutte contre le SIDA. L'UNFPA dans son sixième programme d'assistance au gouvernement du Sénégal a commence aussi à prendre part à cet effort de repositionnement de la SRA dans le Ministère de la Santé.

L'utilisation des résultats de la Recherche Opérationnelle sur l'Amélioration de la Santé de la Reproduction des Adolescent(e)s a permis d'accroître l'intérêt pour la santé des adolescents. Le Sénégal, à travers le Ministère de la Santé et de la Prévention dispose de tous les éléments de base pour l'appropriation et l'extension des activités de santé et de développement des adolescent(e)s. Neuf (9) des onze régions que comptent le Sénégal ont démarré des activités de santé des adolescents selon le modèle de la RO.

Devant ce succès des requêtes ont commencé à parvenir à FRONTIERS pour une assistance technique dans les autres pays. La collaboration avec l'OMS pour cette RO a aussi été l'occasion de développer une stratégie de collaboration avec l'OMS Afro. Des besoins d'appui des autres pays ont été identifiés. Aussi l'USAID a accepté de financer un effort d'expansion vers les autres pays de la sous région en 2007.

\section{OBJECTIFS SPECIFIQUES}

- Promouvoir l'intégration des leçons apprises de la RO, de l'utilisation des résultats de la RO et des outils pour mieux prendre en charge la santé des adolescents dans la communauté des agences de collaboration, des ONGs et des instances gouvernementales dans la région francophone ;

- Renforcer les capacités des institutions de prestation de service à prendre la responsabilité de passer à l'échelle des interventions multisectorielles pour améliorer la santé des adolescents. 


\section{RESULTATS}

\section{Développement d'un partenariat durant un atelier de consultation sur la SRA et partager les expériences du Sénégal avec d'autres pays d'Afrique}

L'OMS qui a été une partie prenante de la Recherche Opérationnelle sur l'Amélioration de la Santé de la Reproduction des Adolescents au Sénégal et dont le bureau du Sénégal est avec FRONTIERES le principal collaborateur du Ministère de la Santé du Sénégal en matière de Santé des Adolescents, a initié avec le programme FRONTIERES une consultation régionale sur les approches communautaires efficaces dans la prise en charge de la santé de la reproduction des Adolescents.

\section{- Contexte favorable}

L'OMS a lancé une approche stratégique nommée APADOC (Alliance of Parents, Adolescents and the Community) pour répondre aux besoins en SRA des adolescents dans neuf pays à savoir le Botswana, l'Ethiopie, le Malawi, le Mozambique, le Swaziland, la Tanzanie, la Zambie et le Zimbabwe). L'objectif de APADOC est d'appuyer les pays dans la gestion intégrée des problèmes majeurs de santé auxquels sont confrontés les adolescents en impliquant les parents, les adolescents et la communauté. Ce modèle se base sur la famille où le processus de socialisation est initié pour soutenir la santé de la reproduction et le développement des adolescents. Les activités mises en œuvre par APADOC visent à renforcer les adolescents par l'information et les compétences sociales et interpersonnelles et à encourager la communication entre parents et adolescents. Un cadre de planification et de mise en œuvre des interventions relatives à la santé des adolescents a été développé dans ces pays. L'outil de base guidant ce processus est la stratégie régionale de l'OMS en Santé de la Reproduction des adolescents. Les ressources ont été identifiées au niveau pays où plusieurs partenaires appuient les programmes verticaux. Les intervenants clés participant à divers stades de ces projets sont les secteurs gouvernementaux pertinents (Santé, Education, Jeunesse, Sport, etc.), les agences des Nations Unies, les ONGs, les parents, les écoles, les communautés et les adolescents. Les leçons tirées de cette initiative doivent elles aussi être partagées avec la région.

UNFPA a aussi conduit une évaluation du projet de l'Alliance Africaine des Jeunes (AYA) qui visait à améliorer la santé sexuelle et reproductive des adolescents à travers une approche multisectorielle dans quatre pays anglophones (Botswana, Ghana, Tanzanie et Uganda). Ce projet a été conçu comme une gamme exhaustive d'interventions intégrées, mise en œuvre simultanément et à l'échelle. Le modèle AYA a six composantes : politique, communication pour un changement de comportement, les services conviviaux pour les adolescents, les programmes de subsistance et le renforcement de capacités institutionnelles. Financé pour une durée de cinq ans, AYA a apporté une innovation dans le renforcement des capacités et nécessite l'appui des gouvernements et autres agences de développement pour le passage à l'échelle des interventions en SRA et en prévention du VIH à l'endroit des jeunes. AYA a crée un héritage de changement de comportement au sein des jeunes touchés directement par le programme et de développement environnement programmatique favorable et durable qui continue d'appuyer la programmation de la SRA dans les quatre pays couverts par AYA. Le projet a eu un impact sur plusieurs indicateurs particulièrement au sein de la gent féminine. 
L'expérience de FRONTIERES au Sénégal et au Kenya et particulièrement leurs expériences pour le passage à l'échelle d'une approche multisectorielle rentrent également dans ce cadre d'échange d'approches holistiques en santé des adolescents.

Ces trois approches apportent de nouvelles visions à la programmation de la santé de la reproduction des adolescents qui sont particulièrement pertinentes maintenant que plusieurs évaluations ont prouvé que les centres pour ados, une stratégie largement utilisée en Afrique, sont coûteux, ne sont pas efficaces et ont un faible impact. En conséquence, il est important de changer le paradigme qui a été jusque là utilisé pour gérer les besoins des adolescents en SRA.

\section{- Partenariat}

FRONTIERES a collaboré avec l'OMS et UNFPA pour jouer un rôle déterminant dans cet effort de promotion de partage des réponses compréhensives et holistiques aux besoins des adolescents en santé de la reproduction et pour encourager l'utilisation de ces leçons apprises de même que les outils, guides et modèles de politique dans des pays francophones choisis.

Toutes ces institutions ont partagé la responsabilité de mettre en œuvre un processus consultatif lors de l'atelier régional :

- Le bureau régional pour l'Afrique de l'OMS a financé la participation des représentants de la Tanzanie, du Mozambique, de la Namibie et du Zimbabwe qui ont mis en œuvre APADOC ;

- UNFPA a appuyé la participation des représentants du Ghana et d'autres pays sélectionnés ;

- FRONTIERES a appuyé la participation des participants du Sénégal, de la Mauritanie, du Kenya et du Burkina Faso et a organisé la présentation des expériences du Sénégal et du Kenya.

- La rencontre qui s'est tenue à Accra au Ghana les 27, 28 et 29 février a réuni des participants venus de l'OMS Genève, de OMS/AFRAO, de Population Council, UNFPA, London School of Hygiene and Tropical Medicine, des Ministères de la Santé, des ONG, des associations de jeunes du Burkina Faso, du Mozambique, de la Tanzanie, du Zimbabwe, de la Mauritanie, du Sénégal, du Ghana et du Kenya. En outre, les missions de l'USAID et CAs, l'UNICEF, la Banque Mondiale et d'autres donateurs ont été invité à participer.

\section{Partage des expériences sur les composantes de la participation communautaire}

L'importance de la participation de la communauté dans les programmes pour adolescents en Afrique subsaharienne a été reconnue par tous les pays participants. En effet, l'absence de cette participation communautaire est longtemps demeuré l'un des points faibles dans la programmation des activités visant les adolescents. Cependant sa mise en œuvre ne maintient pas toujours le lien avec les services de santé.

Suite à la présentation des programmes de jeunes de FRONTIERES, APADOC et AYA, sept pays ont exposé le détail de leurs interventions et les leçons apprises. Les forces et les faiblesses ont été discutées. L'approche et l'expérience de FRONTIERES ont suscité beaucoup d'engouement de la part des autres pays.

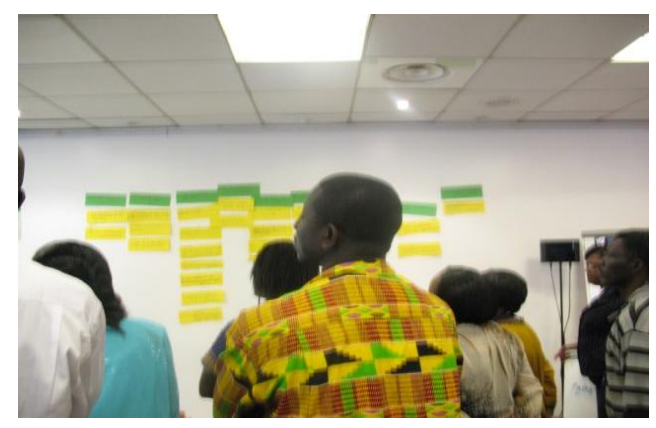


L'observation principale qui est ressortie des discussions était que les projets de FRONTIERES et de AYA ont démontré une pérennité. Cependant le défi reste le maintien de la multisectorialité, particulièrement avec la participation communautaire. Il reste à résoudre la question du suivi et de l'évaluation pour le processus de passage à l'échelle.

D'autres questions centrales sont :

- Les préservatifs comme preuves ou traceur de l'utilisation des services ;

- Les défis majeurs pour l'optention de l'acceptation communautaire du préservatif et son offre aux adolescents ;

- Le contrôle et la mesure de la qualité ;

- Les indicateurs qui montrent l'augmentation du soutien communautaire et la demande des services par les adolescents ;

- L'assurance qualité des interventions ( spécialement si l'engagement de la communauté dans la conception est significative).

\section{Réflexion sur un cadre conceptuel}

Un cadre conceptuel pour les interventions qui doivent mener au soutien de la communauté et à l'acceptation de l'offre et l'utilisation des services de santé pour les adolescents et pour susciter la demande de l'utilisation des services de santé par les adolescents a été débattue (voir ci dessous). La discussion a largement porté sur le fait que les actions pour augmenter la demande peuvent influencer l'offre de services et aussi mettre la pression sur la

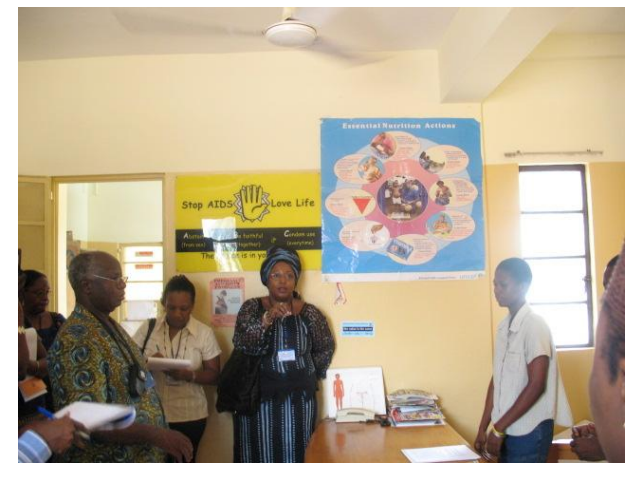
communauté afin qu'elle accepte l'offre et l'utilisation des services.

Interventions pour obtenir l'appui communautaire ou l'acceptation de l'offre de services de santé pour les adolescents et susciter la demande de l'utilisation des services de santé par les adolescents

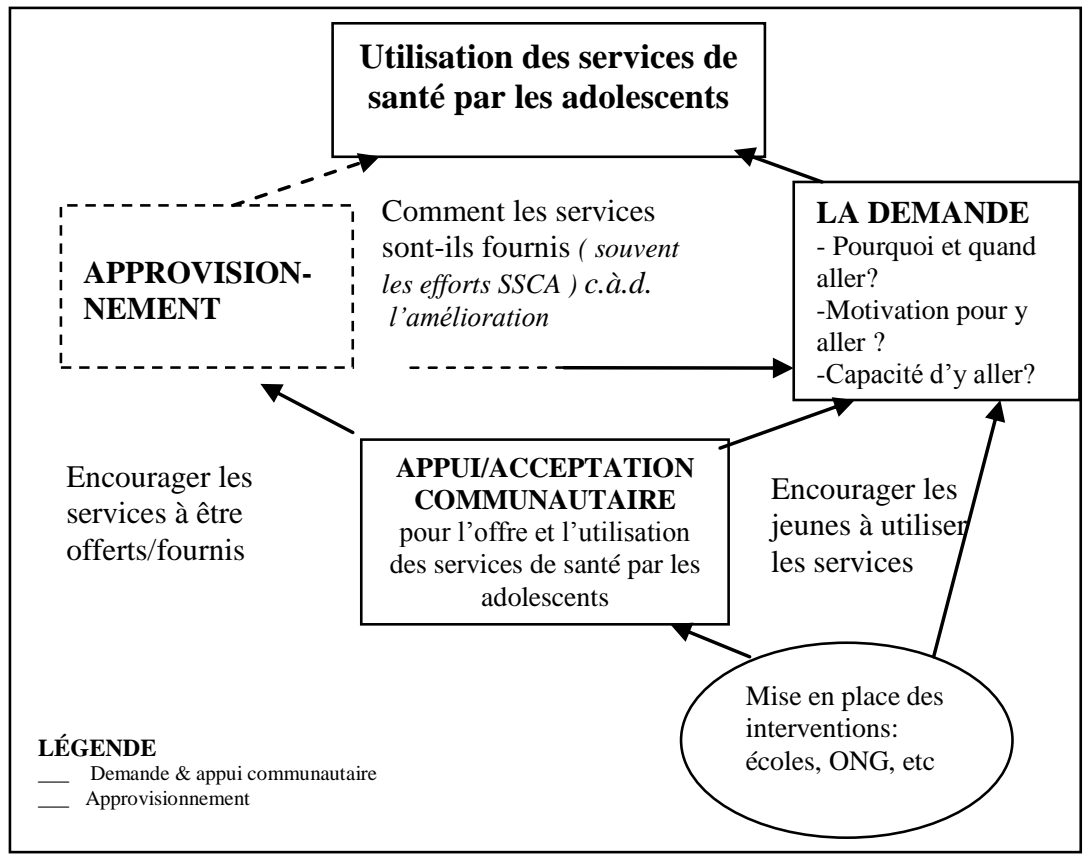


Un certains nombre d'indicateurs ont été identifiés:

- Pour l'appui communautaire/acceptation de l'offre de services et l'utilisation des services :

- Connaissance des besoins des adolescents en services de santé (connaissance)

- Approbation de l'offre de services et l'utilisation (attitude)

- Actions entreprises pour améliorer l'offre de services aux adolescents (exemple à travers le plaidoyer au sein des communautés (comportement)

- Actions entreprises pour améliorer l'utilisation des services par les adolescents (exemple accompagner les adolescents vers les services, octroyer des fonds pour les frais de transport pour se rendre vers ces services (comportement)

- Pour la demande des adolescents en services de santé :

- Les adolescents savent comment, quand et pourquoi les services de santé doivent/peuvent être utilisés (connaissance)

- Les adolescents savent où les services de santé peuvent être obtenus; quelles sont les conditions de leur utilisation (connaissance)

- Les adolescents manifestent leur intention d'utiliser les services de santé au besoin (attitude)

- Les adolescents utilisent(rapportent l'utilisation) les services (comportement)

Développement d'un guide d'orientation pour la programmation de la santé de la reproduction des adolescents

Basée sur les leçons apprises de FRONTIERES et des deux autres programmes, et sur les outils conçus et testés pour chacun d'eux, un aperçu et un format pour un guide générique d'orientation stratégique pour la mise en œuvre d'approches multisectorielles dans la réponse aux besoins en santé des adolescents ont été élaborés pendant l'atelier consultatif. Le guide présente les éléments importants des trois modèles de projets (FRONTIERES, AYA and APADOC).

L'objectif de ce guide est de mettre en relief certains éléments clés dans la programmation multisectorielle de la santé des adolescents qui pourraient être utilisés par les chargés de programme de SRA dans divers secteurs d'activités. Les facteurs favorisants et inhibiteurs ont été identifiés et synthétisés pour une programmation institutionnelle et pour des efforts politiques aux niveaux central et district. Le guide sera générique et chaque pays pourra l'adapter selon ses spécificités.

Les éléments clés pour le guide ont été identifiés à savoir :

- Le cadre conceptuel ;

- Les questions de pérennisation et comment les traiter ;

- Les interventions génériques pertinentes pour l'appui de la communauté et à la demande de services de santé ;

- Quelques indicateurs importants relatifs aux interventions et aux résultats ;

- La collaboration multisectorielle vis-à-vis des groupes cibles (parents, leaders communautaires, les élèves eux-mêmes) ;

- Les considérations liées à la mise en œuvre ;

- La documentation des stratégies qui ont montré leur efficacité ;

- Les messages clefs;

- Les références des ressources matérielles. 
Un comité directeur a été créé pour synthétiser les résultats de l'atelier et rassembler les différentes composantes du guide avec l'appui d'un consultant. Après finalisation, le guide sera disséminé auprès des chargés de programmes et des partenaires au développement à travers l'Afrique.

\section{Renforcement des capacités des institutions offrant des services}

Outre cet effort régional, FRONTIERES a appuyé des activités de renforcement des capacités dans un pays francophone qui a déjà montré son intérêt dans la priorisation des questions de SRA et qui a déjà reçu de l'assistance technique de FRONTIERE afin d'adapter et introduire le modèle du Sénégal.

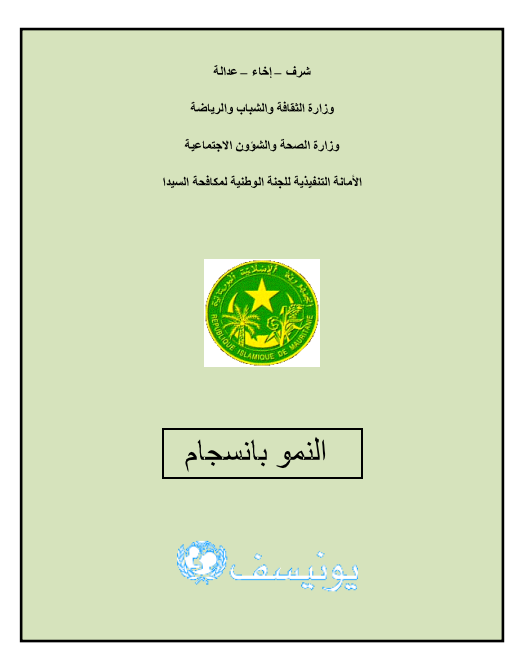

renforcer les capacités des formateurs lors de deux ateliers de formation des formateurs qui ont regroupé 44 éducateurs issus des cinq régions de la Mauritanie. L'UNICEF poursuit le processus et à ce jour près de 200 pairs éducateurs ont été formés à travers le pays avec le curriculum développé lors de la recherche opérationnelle de FRONTIERES.

\section{Le Burkina Faso}

Le Ministère de la Santé du Burkina Faso a déjà pris le leadership dans la réponse aux besoins des adolescents. Il a exprimé un fort intérêt dans l'expérience et les outils du Sénégal suite à la présentation de FRONTIERES lors de l'atelier régional de juin 2005. En plus, il a été convenu que le document de politique du Sénégal sur la stratégie nationale en santé des adolescents offre un modèle pour le Burkina Faso.

Depuis la rencontre de février 2008 à Accra, des discussions avec la Division de la Santé de la Reproduction du Ministère de la Santé du Burkina ont conduit à un partage des outils développés au Sénégal lors des d'un atelier qui devait se tenir à Ouagadougou, mais cette activité n'a pas pu avoir lieu.

\section{Renforcement des capacités par des échanges de formateurs}

Dans le cadre de la RO de FRONTIERES et le processus d'utilisation des résultats et d'institutionnalisation, FRONTIERES a eu à renforcer les capacités de plusieurs centaines de formateurs au Sénégal (voir rapport 1, 2007). Aussi ces formateurs sont prêts à appuyer d'autres 
pays selon les requêtes. FRONTIERES a facilité ce cadre d'échange en demandant l'autorisation du Ministère de la Jeunesse du Sénégal pour l'envoi de formateurs en Mauritanie.

FRONTIERES a financé le déplacement et le séjour de trois formateurs en provenance du Sénégal qui ont résidé durant deux semaines en Mauritanie pour assurer les formations.

FRONTIERES aussi facilité l'échange de formateurs en répondant favorablement à la requête de l'ONG Internationale YMCA. YMCA dispose d'un Centre de Formation Professionnelle, d'un Domaine dans les Niayes et d'un Groupe Scolaire.

Le programme Santé de la Reproduction des Adolescents de YMCA/Sénégal a débuté en 1993 avec le projet inter-ONG Xall Yoon qui regroupait 17 ONG et OCB de jeunes. Avec le soutien de la Fondation Hewlett Packard, YMCA renforce ce programme et met sur place un Comité National composé de professionnels de la Santé et d'une équipe d'animateurs composée de Pairs Educateurs, d'Assistants Animateurs, d'Animateurs, d'assistants formateurs et de formateurs.

En tant que structure d'encadrement national du Conseil National de Lutte contre le SIDA (CNLS) dans le cadre du projet SIDA, YMCA/Sénégal a sollicité l'assistance technique de FRONTIERES pour un renforcement de capacités en Santé de la Reproduction des Adolescent(e)s et VIH/SIDA. FRONTIERES a donc facilité l'échange de formateurs en finançant le voyage et le séjour de trois formateurs venus de Saint-Louis et de Louga.

YMCA a bénéficié du financement de la Banque Mondiale à travers le CNLS pour mener des activités auprès de 200 OCBs de jeunes à travers le Sénégal. Six organisations de jeunes affiliées à YMCA ont entamé un programme d'activités en SRA avec le Curriculum Grandir en Harmonie développé par FRONTIERES touchant ainsi plus de 8000 jeunes (5693 filles et 2626 garçons).

\section{Development d'une collaboration avec Save the Children Suède}

Save the Children Suède est une ONG Internationale dont le bureau régional pour l'Afrique de l'Ouest est basé au Sénégal. Compte tenu de l'impact du VIH/SIDA sur la vie des enfants et du taux d'infection au niveau des jeunes, SCS a décidé d'intégrer la problématique du VIH/SIDA dans son programme régional, à partir de 2004. Ce programme met surtout l'accent sur la prévention de l'infection au niveau des adolescents à travers l'information et l'accès aux services de santé de la reproduction. SCS a identifié certaines ONGs au Togo, au Burkina Faso, au Sénégal, en Guinée et en Côte d'ivoire qui travaillent essentiellement avec des adolescents /jeunes scolarisés et non scolarisés, issus des zones rurales et urbaines ayant entre 10-18 ans.

Ayant identifié le curriculum «Grandir en Harmonie » comme étant un outil très efficace qui peut contribuer à l'atteinte de ses objectifs, SCS a demandé à FRONTIERES 20 exemplaires du curriculum destinés à ses partenaires ONGs en Afrique de l'Ouest. Par ailleurs, SCS est disposée à renforcer la collaboration avec FRONTIERES pour renforcer les capacités des ONG dans la sous région en matière de santé de la reproduction des adolescents. La recherche du financement est en cours.

\section{Partage d'un paquet d'outils et de matériels d'éducation}

L'assistance technique apporté aux différents partenaires et acteurs à travers le renforcement de capacités de leurs agents s'est accompagné de la mise à disposition des curricula de formation Grandir en Harmonie pour les pairs éducateurs et le programme d'orientation des prestataires de santé en santé des adolescents pour les prestataires. Ainsi plus de 200 exemplaires du curriculum ont été distribué, notamment aux OCBs de jeunes tels que YMCA, Synergie Banlieue, le Forum 
du Futur Africain... dont les membres ont été formés, à l'ASBEF (affiliée à l'IPPF), mais aussi à des partenaires au développement tels que Save The Children Suède destinés à ses différents partenaires des pays de la sous région de l'Afrique francophone.

Plusieurs outils de communication développés par FRONTIERES durant la RO ont été reproduits et mis à la disposition des pays, régions et districts pour servir de matériel d'éducation des jeunes. Il s'agit du déliant Savoir pour Agir reproduit en 1000 exemplaires, du petit carnet de bonne conduite reproduit en 1000 exemplaires et de 300 CD-Rom du curriculum. Des pochettes sur la santé des adolescents permettant de contenir du matériel d'information et de promotion a aussi été reproduit en 100 exemplaires et disséminé.

\section{Augmentation par les média de l'attention des leaders politiques, des responsables de programmes et du public sur la sante des adolescents}

Un des nouveaux défis a été l'utilisation des médias régionaux pour atteindre des pays de la sous région à travers des émissions radiophoniques. C'est dans ce cadre qu'une série d'émission est en cours avec la West African Democracy Radio (WADR) sur les questions touchant les jeunes de façon générale, et leur santé en particulier. La WADR a été créée par OSIWA que veut offrir aux Africain un canal d'échange d'informations entre eux et établir une interaction avec le reste du monde. C'est une radio bilingue transterritoriale qui a pour objectif de favoriser l'échange d'informations sur le développement dans les pays de l'Afrique de l'Ouest ainsi qu'au Tchad, au Cameroun et en Mauritanie. Elle a son siège à Dakar au Sénégal et un bureau ouvert dans chacun des trois pays membres de l'Union du Fleuve Mano (Guinée, Libéria et Sierra Léone). C'est donc une radio de transmission qui se veut être le croisé entre les réseaux publics, privés et les radios communautaires dans la sous région. Ces émissions sont animées par des personnes ressources des différents Ministères et des différents acteurs de la santé et du développement des adolescent à travers les trois canaux qu'offrent la radio dans son programme hebdomadaire :

- Capital Santé (Lundi)

- Fifty-Fifty (Mercredi sur les questions de genre)

- Réseau jeunesse (Vendredi)

FRONTIERES a donc développé un partenariat avec cette radio pour une série d'émissions sur la santé des adolescents. Les évidences des recherches, les leçons apprises des interventions, les défis liés au passage à l'échelle servent de sujet de discussion entre les journalistes, FRONTIERES et certains partenaires du gouvernement et des ONGs.

\section{DIFFICULTES}

Plusieurs problèmes ont entravé la pleine mise en œuvre de ce travail.

- Problème de suivi et de leadership dans les secteurs ministériels de la Mauritanie et du Burkina Faso;

- Problème de lenteur dans l'exécution de l'activité régionale avec l'OMS afin d'influencer les autres pays de la sous région;

- Problème de financement pour assurer la pérennisation des programmes de SRA. 


\section{CONCLUSION}

Une des leçons apprises de cette phase est que l'investissement à long terme pour le plaidoyer et l'assistance technique est essentiel pour créer un environnement politique favorable à la santé des adolescents. La communauté et les gouvernements sont conscients de la nécessité de prendre en charge la santé de la reproduction des adolescents. Les interventions multisectorielles impliquant les ministères, la communauté, les leaders religieux, les familles et les adolescents eux mêmes tels que testés par FRONTIERES ont montré leur efficacité pour l'amélioration des connaissances et des comportements en matière de santé de la reproduction.

Il existe un grand besoin d'assistance technique au niveau des pays pour la santé des adolescents. Le processus utilisé par FRONTIERES pour influencer les programmes en Afrique est certainement une bonne approche. Cela a permis de toucher des partenaires au développement tels que l'OMS, UNFPA, Save The Children Suède, mais aussi plusieurs pays francophones et anglophones. Cette approche multisectorielle devrait devenir une approche régionale si l'on veut arriver à changer les paradigmes qui ont été jusque là utilisé sans résultats probants et arriver à une réponse exhaustive aux questions de santé sexuelle et reproductive des adolescents et des jeunes dans les pays africains. 


\section{ANNEXE 1}

\section{REGISTRATION FORM \\ REGIONAL CONSULTATION ON COMMUNITY BASED INTERVENTION TO IMPROVE THE USE OF HEALTH SERVICES BY ADOLESCENTS \\ ACCRA, GHAHA, 27-29 FEBRUARY 2008}

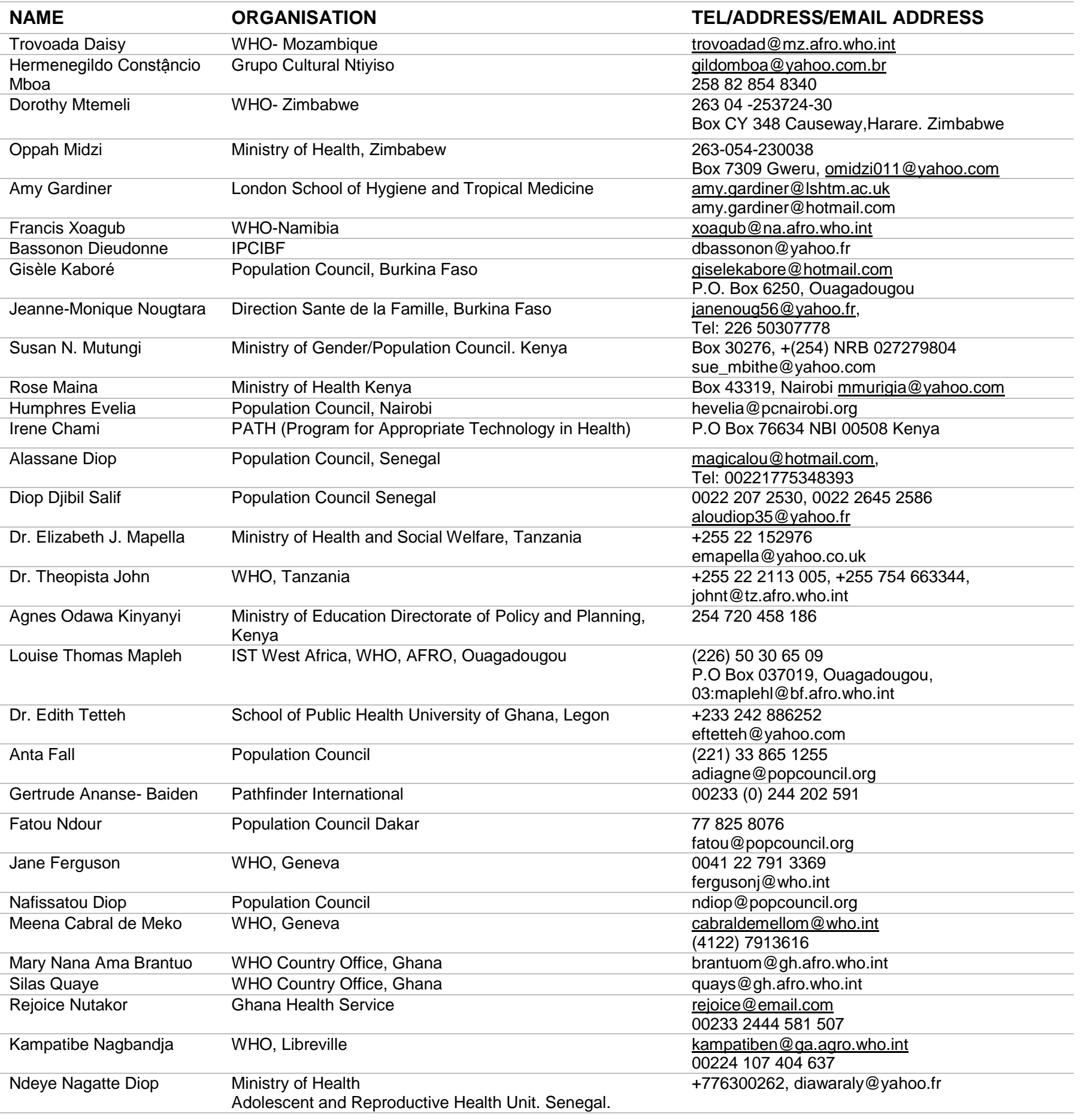

